\title{
A prospective seroepidemiological study of toxocariasis during early childhood in coastal Ecuador: potential for congenital transmission and risk factors for infection
}

Aida Y. Oviedo-Vera ${ }^{1,2}$, Irina Chis Ster ${ }^{3}$, Martha E. Chico ${ }^{1}$, Marcia B. Silva², Luis F. Salazar-Garcés ${ }^{1,2}$, Neuza M. Alcantara-Neves ${ }^{2}$ and Philip J. Cooper ${ }^{1,3,4^{*}}$ (D)

\begin{abstract}
Background: Although Toxocara spp. infection has a worldwide distribution, to our knowledge, no data from birth cohorts have been reported in published studies on the potential for congenital transmission and determinants of infection in early childhood.

Methods: We followed 290 mother-infant pairs from birth to 5 years of age through periodic collection of data and samples at birth, 7 and 13 months and 2, 3 and 5 years of age. Data on potential risk factors and confounders were collected by maternal questionnaire. Blood for plasma was collected from the mother at time of birth and periodically from the child for detection of anti-Toxocara spp. immunoglobulin G (IgG) antibodies using a Toxocara canis larval excretory-secretory antigen-based enzyme-linked immunosorbent assay. Stool samples were collected from the mother around the time of birth and periodically from the child for microscopic detection of soil-transmitted helminths (STH). Associations between potential risk factors and Toxocara spp. seroprevalence and seroconversion were estimated using multivariable logistic regression and generalized estimating equations.
\end{abstract}

Results: Toxocara spp. seroprevalence was $80.7 \%$ in mothers and in children was $0 \%, 9.3 \%, 48.4 \%, 64.9 \%$, and $80.9 \%$ at 7 months, 13 months, 2, 3 and 5 years, respectively. Risk factors significantly associated with increases in seroprevalence over the first 5 years of life in multivariable analyses were age [Odds ratio (OR) 2.06, 95\% confidence interval (Cl) 1.39-2.27, $P<0001$ ], male sex (female vs. male: OR 0.66, 95\% Cl 0.48-0.89, $P=0.006$ ), maternal ethnicity (non-Afro vs. Afro-Ecuadorian: OR $0.65,95 \% \mathrm{Cl} 0.47-0.91, P=0.011$ ), lower maternal educational and socioeconomic level, and childhood STH (OR 2.29, 95\% Cl 1.51-3.47, $P<0.001)$. Seroconversion rates for infection were greatest at 2 years of age (3.8\%/month). Factors associated significantly with seroconversion at 2, 3 or 5 years were childhood STH infection, male sex, and more frequent domestic cat exposure.

Conclusions: Our data, from an area of high Toxocara spp. endemicity, indicate no congenital transmission but high rates of seroconversion after 13 months of age reaching maternal levels of seroprevalence by 5 years of age. Factors associated with seroprevalence and seroconversion included STH infections, domestic cats, maternal ethnicity, male sex, STH infections, and markers of greater poverty.

*Correspondence: pcooper@sgul.ac.uk

${ }^{3}$ Institute of Infection and Immunity, St George's University of London,

Cranmer Terrace, London SW17 ORE, UK

Full list of author information is available at the end of the article

c) The Author(s) 2021. This article is licensed under a Creative Commons Attribution 4.0 International License, which permits use, sharing, adaptation, distribution and reproduction in any medium or format, as long as you give appropriate credit to the original author(s) and the source, provide a link to the Creative Commons licence, and indicate if changes were made. The images or other third party material in this article are included in the article's Creative Commons licence, unless indicated otherwise in a credit line to the material. If material is not included in the article's Creative Commons licence and your intended use is not permitted by statutory regulation or exceeds the permitted use, you will need to obtain permission directly from the copyright holder. To view a copy of this licence, visit http://creativeco mmons.org/licenses/by/4.0/. The Creative Commons Public Domain Dedication waiver (http://creativecommons.org/publicdomain/ zero/1.0/) applies to the data made available in this article, unless otherwise stated in a credit line to the data. 
Keywords: Toxocara spp., Birth cohort, Congenital transmission, Seroprevalence, Seroconversion, Risk factors, Childhood, Ecuador

\section{Background}

Toxocariasis is a common parasitic disease with a worldwide distribution [1]. Toxocara spp. infection affects particularly children in tropical regions living in conditions of poverty and poor hygiene among whom seroprevalence rates often exceed 50\% [1]. Toxocara spp. include Toxocara canis and Toxocara cati that parasitize the small intestine of dogs and cats, respectively. Humans and several animals are paratenic hosts, infected through accidental ingestion of embryonated eggs through contaminated water, soil, and food. Toxocara spp. cannot develop in humans beyond the larval stage, although larvae may survive migrating in tissues for months or years [1].

While most human infections are asymptomatic, infection may have severe clinical consequences causing visceral larval migrans or infections of the central nervous system including the eyes [1]. Disease severity is considered to depend on larval burden and host inflammatory response to invasive larvae [1].

It has been suggested that congenital infections may arise from larval invasion of the placenta from a parasitized mother $[2,3]$. However, there is no definitive evidence of congenital transmission in humans, although congenital infections have been demonstrated in other paratenic hosts such as mice [4]. There are few data documenting the epidemiology of toxocariasis from birth in human populations [1]. We used data and samples from a birth cohort in Ecuador to study the potential for congenital transmission and determinants of seroprevalence and seroconversion in early childhood.

\section{Methods}

\section{Study design}

The present study was nested within a larger birth cohort, the ECUAVIDA cohort, of 2404 mother-child pairs followed from birth to 5 years of age in the district of Quinindé in Esmeraldas Province, Ecuador, that has been described in detail elsewhere [5]. Quinindé District, which is largely rural, is in a tropical region of coastal Ecuador (Fig. 1), and has a mean annual temperature of $30{ }^{\circ} \mathrm{C}$ and relative humidity of $80 \%$; the district has a population of 88,000 inhabitants, who live below $150 \mathrm{~m}$ altitude. Main sources of income are cultivation of palm oil and tropical fruits, and timber extraction. Motherchild pairs for the analysis were selected on the basis of availability of a maternal blood and cord blood sample. Data and samples were collected at around the time of birth of the child and at 7 and 13 months, and 2, 3 and 5 years.

\section{Data and sample collection}

Risk factor data were collected by maternal questionnaire, blood samples were collected for plasma, and stool samples were collected for parasite microscopy using a combination of direct saline wet mounts, modified Kato-Katz, and formol-ether concentration methods [6]. Anthelmintic treatment was provided to mothers with soil-transmitted helminth (STH) infections after delivery and to children with positive stools for STH parasites, as described [6]. Toxocara spp. immunoglobulin G (IgG) antibodies were measured in plasma using a standardized in-house indirect enzyme-linked immunosorbent assay with T. canis larvae excretory-secretory antigens, and a pre-adsorption step against Ascaris lumbricoides antigens, as described [7]. A pool of sera from 10 Toxocara spp.-infected subjects was used as the positive control and a pool of 14 Toxocara spp.-uninfected subjects (i.e. children without history of contact with dogs and cats) was used as the negative control. The cut-off for positivity was the mean optical density of negative control wells +3 SDs.

\section{Statistical analysis}

For statistical analysis, Toxocara spp. seropositivity, defined as a binary longitudinal repeated-measures outcome, was investigated for potential associations with relevant covariates, some of which were time-varying (i.e. cat and dog exposures in the child's household and STH infections). Population average models were fit to these binary outcomes using generalized estimating equations (GEE), as described $[8,9]$, with the assumption of missing at random. Seroconversion rates (\%) of infection were determined for each time period between sampling as the proportion of seronegative children who became positive. Logistic regression models were used to explore associations between seroconversion rates at 2, 3 and 5 years and potential risk factors in preference to a single GEE model that gave unreliable estimates. For logistic regression models, cumulative exposures to domestic pets or STH up to the analysis time point were used as covariates. Multivariable models were constructed with backward elimination from an initial model containing 
all variables with $P<0.1$ in univariate analyses. Statistical significance was inferred by $P<0.05$. Analyses were done using Stata version 11.

\section{Results}

Plasma samples were analysed for 290 mothers and their children at birth (cord blood, 290/290), for 267 (92.1\%) samples collected at 7 months, and for 267 (92.1\%), 261 (90.0\%), 268 (92.4\%), and 247 (85.2\%) samples collected at 13 months, and 2, 3 and 5 years, respectively. Seropositivity for Toxocara spp. IgG was $80.7 \%$ in mothers, $84.1 \%$ in cord blood, and $0 \%$ at 7 months, $10.9 \%$ at
13 months, $49.0 \%$ at 2 years, $65.7 \%$ at 3 years, and $81.0 \%$ at 5 years (Fig. 2). Distributions of potential risk factors and frequencies of Toxocara spp. seropositivity at 2, 3 and 5 years are shown in Table 1 . Overall crude and adjusted associations with potential risk factors over the observation period are shown also in Table 1. Variables significantly associated with seroprevalence in adjusted analyses were: age [Odds ratio (OR) 2.06, 95\% confidence interval (CI) 1.66-2.56, $P<0.001$ ], male sex (female vs. males, OR 0.66, 95\% CI 0.48-0.89, $P=0.006$ ), maternal ethnicity (non-Afro-Ecuadorian vs. Afro-Ecuadorian, OR $0.65,95 \%$ CI $0.47-0.91, P=0.011)$, lower maternal

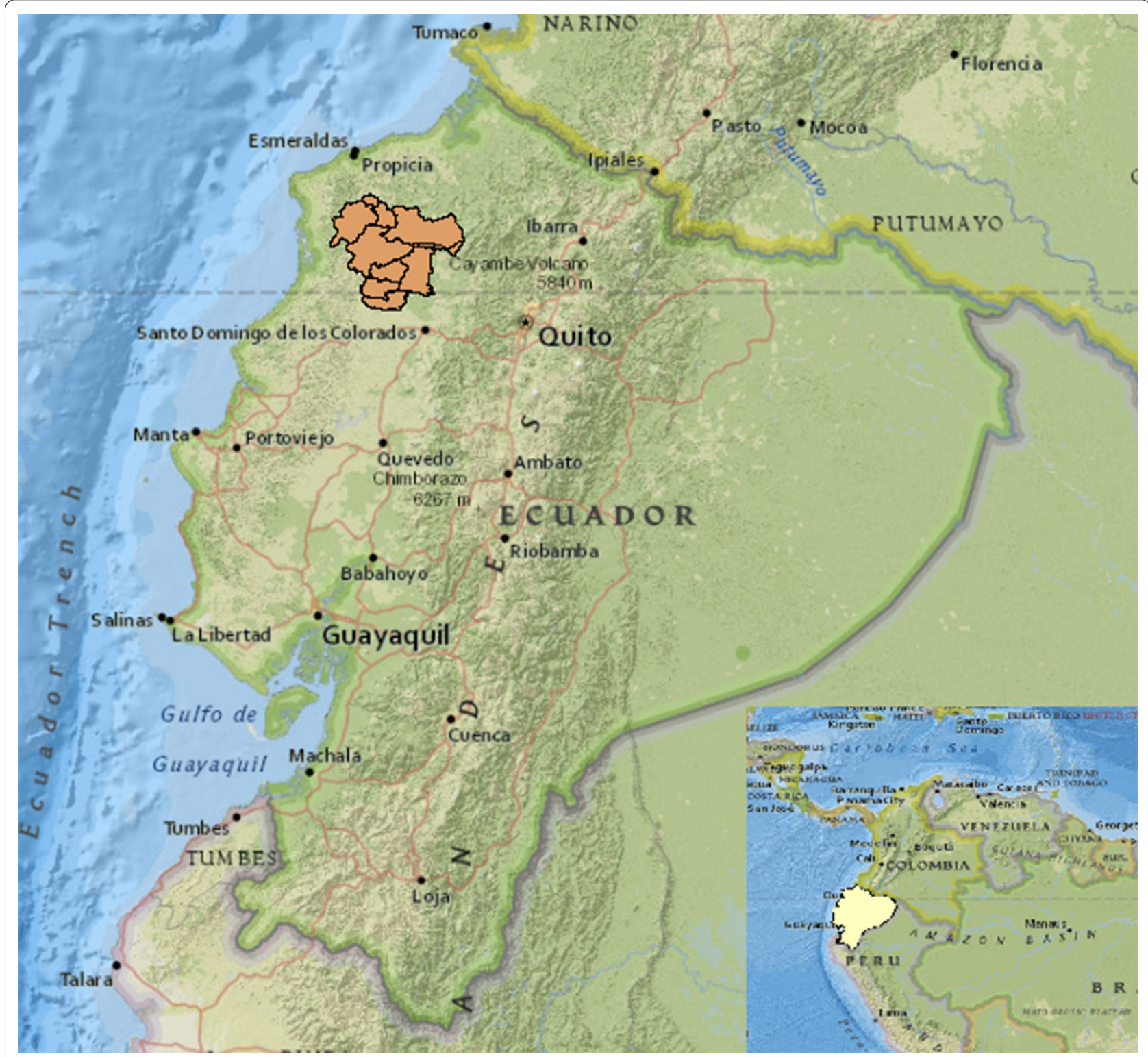

Fig. 1 Map of the study area in the district of Quinindé, Esmeraldas Province, Ecuador 
educational level (completed primary vs. illiterate, OR 0.61 , 95\% CI $0.40-0.93, P=0.021$; completed secondary vs. illiterate, OR $0.57,95 \% \mathrm{CI} 0.34-0.96, P=0.034$ ), socioeconomic level (average vs. low, OR 0.62, 95\% CI $0.43-0.89, P=0.010$; high vs. low, OR 0.60, 0.40-0.89, $P=0.009$ ), and a childhood STH infection (OR 2.29, 95\% CI 1.51-3.47, $P<0.001)$. Variables included in the multivariable model but not reaching statistical significance were maternal Toxocara spp. seropositivity (OR 1.46, 95\% CI 0.97-2.13, $P=0.064$ ) and household cats (OR $1.40,95 \%$ CI $0.99-1.97, P=0.055)$. Seroconversion rates were: $10.9 \%(29 / 267)$ between 7 and 13 months, $42.2 \%$ (95/225) between 13 months and 2 years, 35.1\% (47/134) between 2 and 3 years, and 45.7\% (37/81) between 3 and 5 years. Seroconversion rates per month/years were $1.8 \%$, $3.8 \%, 2.9 \%$, and $1.9 \%$ at 13 months, 2,3 and 5 years of age, respectively (Fig. 3). Few children reverted to seronegative once seropositive: none of 24 children seropositive at 13 months became negative at 2 years (among children for whom results were available at both 13 months and 2 years), 3 of 128 (2.3\%) positive children at 2 years became negative at 3 years, and 3 out of $176(1.7 \%)$ positive children at 3 years were negative at 5 years. Significant determinants of seroconversion in adjusted analyses were: between 13 months and 2 years, having any STH infection [adjusted (adj.) OR 2.80, 95\% CI 1.38-5.70, $P=0.004$ ]; between 2 and 3 years, male sex (female vs. male, adj. OR $0.37,95 \%$ CI $0.17-0.78, P=0.009)$ and more frequent reports of domestic cats $(>=4$ reports vs. $0-1$ report, adj. OR 5.20, 95\% CI 1.13-23.89, $P=0.034$ ); and between 3 and 5 years, any STH infection (adj. OR $3.46,95 \%$ CI $1.22-9.81, P=0.019$ ).

\section{Discussion}

In the present analysis, we used a birth cohort recruited in a rural district in a tropical region of coastal Ecuador to address two questions relating to the epidemiology of human toxocariasis: whether infection can occur through congenital transmission, and what are the risk factors for the acquisition of infections during childhood. We followed a sample of 290 newborns longitudinally until 8 years of age and showed that at 7 months of age none of the children studied had evidence of anti-Toxocara spp. IgG antibodies, indicating that congenital transmission from seropositive and presumably infected mothers is unlikely to occur in this setting. The acquisition of antiToxocara spp. IgG antibodies was associated with childhood STH infections, male sex, maternal ethnicity, and household poverty.

We observed a high seroprevalence for toxocariasis in our study population of approximately $80 \%$ in mothers and in children by 5 years of age. The seroprevalence

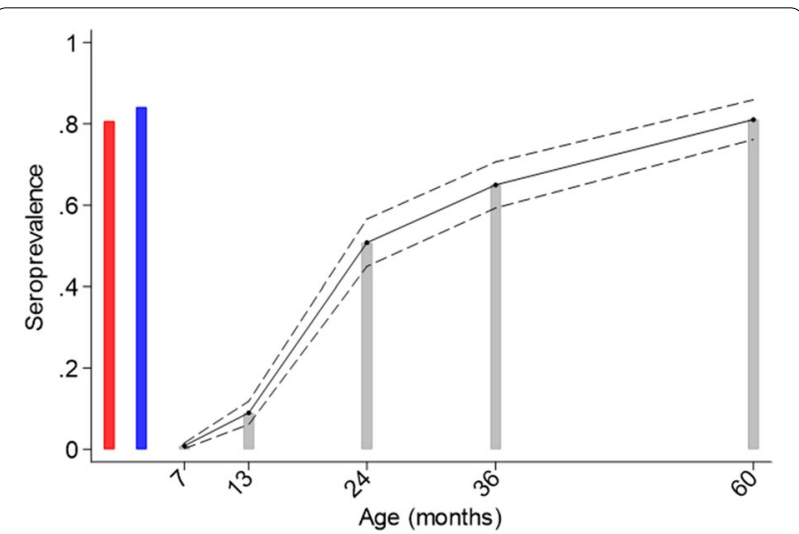

Fig. 2 Anti-Toxocara spp. immunoglobulin G seroprevalence in mothers and in children from birth to 60 months of age. Shown are results from mothers during the third trimester of pregnancy (red bar), cord blood (blue bar) and children at 7, 24, 36, and 60 months (grey bars) of age. Actual (bars) and predicted (continuous line with $95 \%$ confidence intervals as dashed lines) seroprevalence are shown

observed in this population is as at the upper level of that observed previously in other low and middle-income countries [10]. While seropositivity does not imply active infection-Toxocara spp. larvae have been estimated to survive in a human host for 2-4 years [11-13]-the fact that few children reverted to seronegative over the course of the study $(<2.5 \% /$ year $)$ indicates that reinfections were common in this setting.

Vertical transmission of toxocariasis as a consequence of larval migration via the placenta has been suggested to occur following exposure to infection during pregnancy or through re-activation of dormant larvae in a chronically infected woman undergoing the immunological changes associated with pregnancy [2]. The only previous report of potential in utero transmission in humans was in a newborn of a seropositive mother, who developed a Toxocara-like lesion in the eye, which was detected by ultrasound and disappeared after chemotherapy [3]. Although highly suggestive, the latter report does not provide unequivocal evidence for congenital transmission: definitive diagnosis requires detection of larvae or larval DNA in tissue or body fluid samples. Transmission of T. canis in dogs, a natural host, is primarily through transplacental and transmammary transmission [4], with re-activation of dormant larvae occurring during pregnancy [14]. Congenital transmission has been documented also in paratenic hosts such as mice through sequestration of larvae in the brain [4]. In the present study two observations did not favour congenital transmission: although seropositivity in cord blood was equivalent to that in mothers (representing transplacental transfer of maternal IgG to foetus), anti-Toxocara spp. IgG 


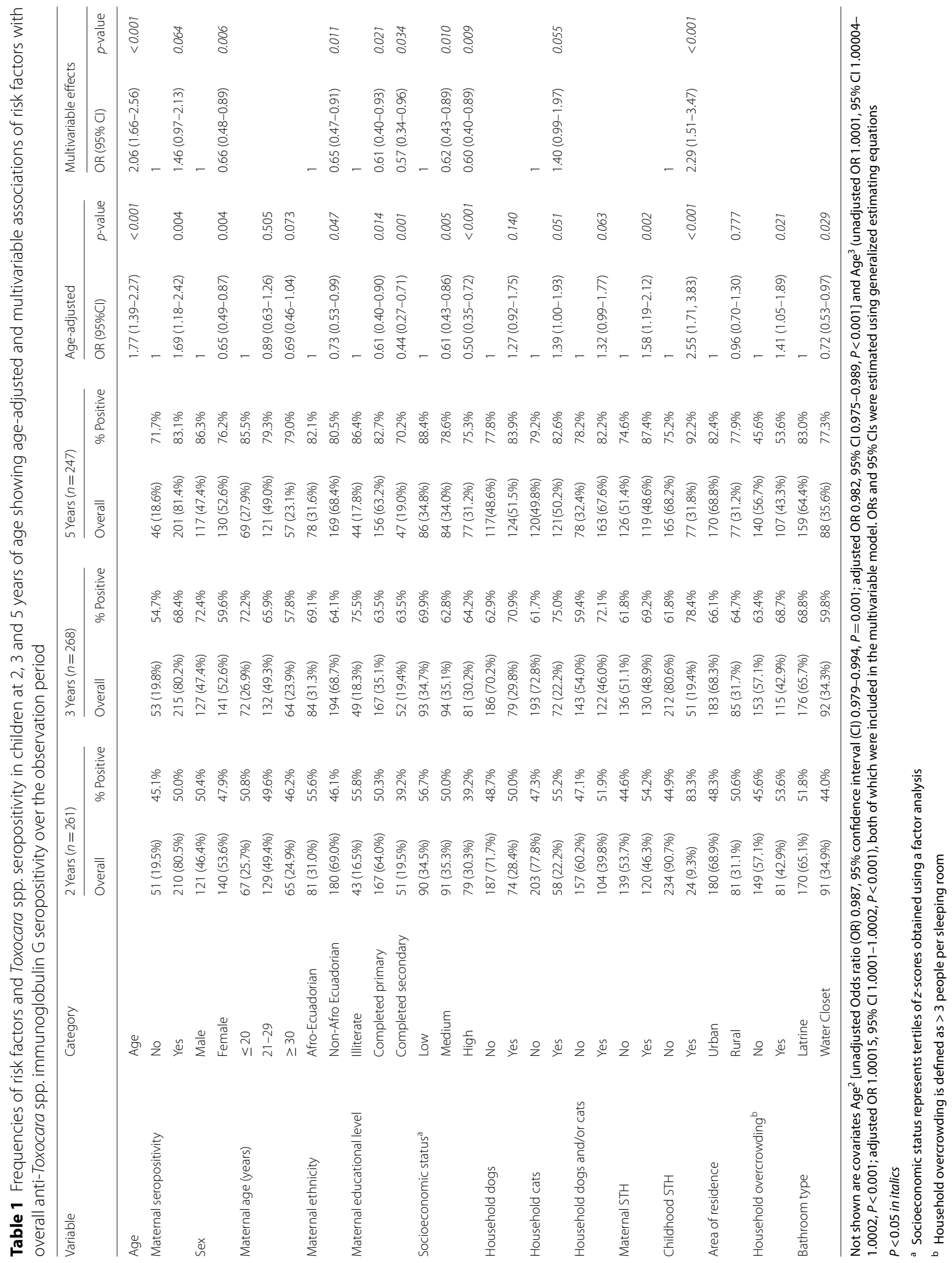




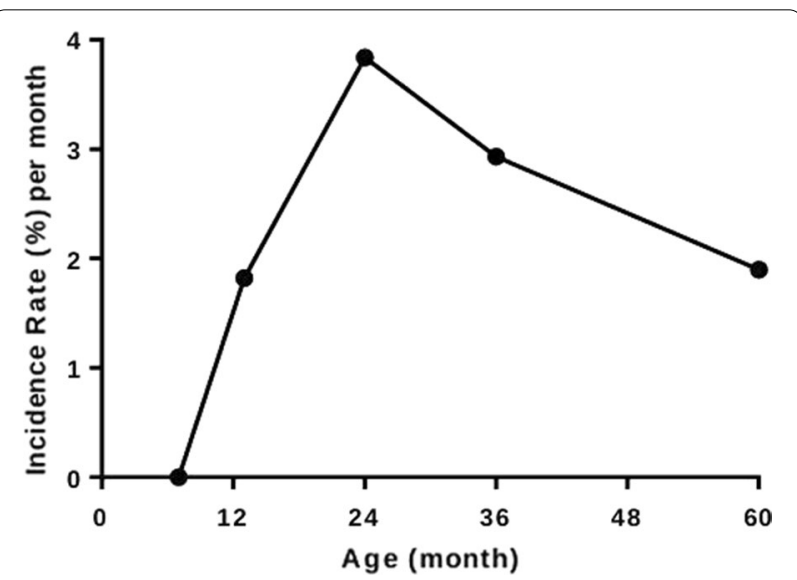

Fig. 3 Monthly seroconversion rates at 7, 13, 24, 36 and 60 months of age

antibodies became undetectable by 7 months of age in all children as the infants cleared maternal antibodiesafter 7 months, seroconversion occurred rapidly and reached maternal levels of seropositivity by 5 years of age; maternal seropositivity was not strongly associated with seroprevalence or seroconversion in the cohort.

The few previous longitudinal studies on children that estimated seroconversion rates for anti-Toxocara spp. IgG antibodies observed cohorts of children for 1 year: (1) a study in Campinas in São Paulo State in Brazil, done in a population with $28 \%$ seroprevalence among children, followed 72 seronegative children aged 6-14 years and estimated an incidence of $7.6 \%$ (or $0.6 \% /$ month) [15]; (2) another study from Campinas, done in a population with $14.6 \%$ seroprevalence, followed 77 seronegative children aged 2-12 years and observed a seroconversion rate of $10.4 \%$ (or $0.9 \% /$ month) [13]; and (3) a study in the city of Assis Brazil, in Acre State in Brazil [16], done in a population with $24 \%$ seroprevalence in children, followed 228 children aged 6 months to 12 years and observed seroconversion rates of up to $13.9 \%$ (or $1.2 \%$ /month) depending on the age of the children [16]. These seroconversion rates are slightly lower than those observed in the present study (average of $1.3 \%$ /month), in which we observed children over a 5-year period from birth. However, in the present study, the risk of acquiring infection was greatest during the second (3.8\% seroconversion rate/month) and third $(2.9 \% /$ month $)$ years of life.

Toxocara spp. infections during early childhood are likely to be acquired in and around the household. Sources of infection are domestic dogs and cats [11, 17$19]$ in the context of poor hygiene and open contamination of the peri-domestic environment with pet faeces [20]. Infected pet faeces can contaminate play areas of young children and drinking water of households without potable water [16]. Peri-domestic environments are often more heavily contaminated with Toxocara spp. eggs than recreational areas (e.g. parks and public play areas) $[20,21]$. Male sex is a recognized risk factor in many settings $[11,17,18]$, and is likely a reflection of genderdetermined patterns of play, as are co-infections with STH through shared risk factors of poor environmental hygiene $[7,17,22]$. We have documented previously, in a different population in coastal Ecuador, the presence of Toxocara spp. DNA in floor and mattress dust samples taken inside households [23] -mattress samples were positive more frequently than floor samples, and the presence of $T$. canis DNA was more frequently detected than T. cati DNA ( $23 \%$ vs. $4 \%$ of samples, respectively) [23]. Contamination of beds could occur through pets grooming themselves in sleeping areas: infection rates among cats may be very high in some settings [24], and embryonated Toxocara spp. eggs can be detected in pet fur at much higher densities than found in soil [25-28]. The relative importance of dog versus cat transmission in a specific setting may vary by infection rates among pets and local customs with respect to entry of pets into houses. In the present study, exposure to cats was a nonsignificant risk factor for seroprevalence, while cumulative cat exposures, but not dog exposures, were strongly associated with seroconversion in children between 2 and 3 years of age.

This study had a number of important limitations. We inferred infection by the presence of anti-Toxocara spp. IgG antibodies using a well-validated serological assay. Such assays may be less specific in populations exposed to other helminth infections such as A. lumbricoides with which Toxocara spp. share significant immunologic cross-reactivity [1,7]. Despite pre-adsorption of test sera against a phosphate buffered saline extract of $A$. lumbricoides adult worms to minimize serological cross-reactions [7], we cannot exclude false positive reactions relating to other $\mathrm{STH}$ co-infections. Use of assays such as Western blot to improve specificity is useful for individual diagnosis but less practical for epidemiological surveys. Although childhood STH infections were an important risk factor for Toxocara spp. seropositivity, this observation is perhaps most likely explained by shared risk factors and uncontrolled confounding. The sample size was relatively small, limiting our ability to identify risk factors with relatively small effects and infrequent outcomes such as allergy. The sample was sufficient, however, to show that congenital transmission, if it does occur, is an unusual occurrence in this population (i.e. $<1$ in 200). Important strengths were the longitudinal design from birth, frequent sampling and data collection allowing age-dependent seroconversion rates to be estimated, the collection of data on a wide range 
of potential risk factors and confounders, and high rates of follow up in excess of $85 \%$ at all sampling time points which would have minimized selection bias.

\section{Conclusions}

In conclusion, to our knowledge, this is the first study to use a birth cohort to examine the acquisition of anti-Toxocara spp. IgG antibodies during the first 5 years of life in an area of high endemicity in a tropical setting. Our data suggest that if congenital transmission does occur in this setting it is a rare occurrence, and that important risk factors for acquisition of infection are household cats, male sex, and concurrent STH infections indicating poor hygiene.

\begin{abstract}
Acknowledgements
We thank the ECUAVIDA study team for their dedicated work and the cohort mothers and children for their enthusiastic participation in the study. We acknowledge also the support of the directors and staff of the Padre Alberto Buffoni Hospital in Quininde, Esmeraldas Province. The study forms part of the Social Changes, Asthma and Allergies in Latin America programme of research. The graphical abstract was drawn by Elizabeth Cooper, and the map was provided by Dr Alejandro Rodriguez.
\end{abstract}

\section{Authors' contributions}

PJC and NMAN designed and supervised the study. PJC and AYO drafted the manuscript. AYO, MBS and LFS did the sample analyses. ICS and PJC did the statistical analyses. MEC supervised the collection of data and samples. All authors read and approved the final manuscript.

\section{Funding}

The study was funded by the Wellcome Trust (grant 088862/Z/09/Z).

\section{Availability of data and materials}

Data are available upon request.

\section{Ethics approval and consent to participate}

The study protocol was approved by the Ethics Committees of the Pedro Vicente Maldonado Hospital and Universidad San Francisco de Quito, Quito, Ecuador. The study was registered as an observational study (ISRCTN 41239086). Informed written consent was obtained from the mothers for their participation and that of their children.

\section{Consent for publication}

Not applicable.

\section{Competing interests}

The authors declare that they have no competing interests.

\section{Author details \\ ${ }^{1}$ Fundacion Ecuatoriana Para Investigación en Salud, Quinindé, Ecuador. ${ }^{2}$ Institute of Health Sciences, Federal University of Bahia, Salvador, Bahia, Brazil. ${ }^{3}$ Institute of Infection and Immunity, St George's University of London, Cranmer Terrace, London SW17 ORE, UK. ${ }^{4}$ Escuela de Medicina, Universidad Internacional del Ecuador, Quito, Ecuador.}

Received: 2 November 2020 Accepted: 29 December 2020 Published online: 05 February 2021

\section{References}

1. Ma G, Holland CV, Wang T, Hofmann A, Fan C-K, Maizels RM, et al. Human toxocariasis. Lancet Infect Dis. 2018;18:14-24.
2. Anderson $B C$. Warning about potential for congenital neural larva migrans. J Am Vet Med Assoc. 1996;208:185.

3. Maffrand R, Avila-Vázquez M, Princich D, Alasia P. Congenital ocular toxocariasis in a premature neonate. An Pediatr (Barc). 2006;64:599-600.

4. Schoenardie ER, Scaini CJ, Pepe MS, Borsuk S, Avila LF, Villela M, et al. Vertical transmission of Toxocara canis in successive generations of mice. Rev Bras Parasitol Vet. 2013;22:623-6.

5. Cooper PJ, Chico ME, Platts-Mills TAE, Rodrigues LC, Strachan DP, Barreto ML. Cohort profile: the Ecuador life (ECUAVIDA) study in Esmeraldas Province, Ecuador. Int J Epidemiol. 2015;44:1517-27.

6. Cooper PJ, Chico ME, Vaca MG, Sandoval CA, Loor S, Amorim LD, et al. Effect of early-life geohelminth infections on the development of wheezing at 5 years of age. Am J Respir Crit Care Med. 2018;197:364-72.

7. Mendonça L, Veiga R, Dattoli V, Figueiredo C, Fiaccone R, Santos J, et al. Toxocara seropositivity, atopy and wheezing in children living in poor neighbourhoods in urban latin American. PLoS Negl Trop Dis. 2012;6:1-9.

8. Zeger SL, Liang K-Y, Albert PS. Models for longitudinal data: a generalized estimating equation approach. Biometrics. 1988:44:1049-60.

9. Zeger SL, Liang KY. An overview of methods for the analysis of longitudinal data. Stat Med. 1992;1 1:1825-39.

10. Fialho PMM, Corrêa CRS. A systematic review of toxocariasis: a neglected but high-prevalence disease in Brazil. Am J Trop Med Hyg. 2016;94:1193-9.

11. Buijs J, Borsboom G, Renting M, Hilgersom WJA, Van Wieringen JC, Jansen $\mathrm{G}$, et al. Relationship between allergic manifestations and Toxocara seropositivity: a cross-sectional study among elementary school children. Eur Respir J. 1997;10:1467-75.

12. Elefant GR, Shimizu SH, Arroyo Sanchez MC, Abe Jacob CM, Ferreira AW. A serological follow-up of toxocariasis patients after chemotherapy based on the detection of $\operatorname{lgG}$, IgA, and IgE antibodies by enzyme-linked immunosorbent assay. J Clin Lab Anal. 2006;20:164-72.

13. Fialho PMM, Correa CRS, Lescano SZ. Asthma and seroconversion from Toxocara spp. infection: which comes first? Biomed Res Int. 2018;2018:1-6.

14. Burke TM, Roberson EL. Prenatal and lactational transmission of Toxocara canis and Ancylostoma caninum: experimental infection of the bitch at midpregnancy and at parturition. 1985;15:485-90

15. Correa CRS, Bismarck CM. Toxocariasis: incidence, prevalence and the time serum remains positive in school children from Campinas, SP, Brazil. J Trop Pediatr. 2010;56:215-6.

16. Oliart-Guzmán H, Delfino B, Martins A, Mantovani S, Braña A, Pereira $T$, et al. Epidemiology and control of child toxocariasis in the western Brazilian Amazon - a population-based study. Am J Trop Med Hyg. 2014;90:670-81.

17. Mendonça LR, Figueiredo CA, Esquivel R, Fiaccone RL, Pontes-deCarvalho L, Cooper P, et al. Seroprevalence and risk factors for Toxocara infection in children from an urban large setting in northeast Brazil. Acta Trop. 2013;128:90-5.

18. Silva MB, Amor ALM, Santos LN, Galvão AA, Oviedo VAV, Silva ES, et al. Risk factors for Toxocara spp. seroprevalence and its association with atopy and asthma phenotypes in school-age children in a small town and semirural areas of northeast Brazil. Acta Trop. 2016;174:425-8.

19. Raissi V, Taghipour A, Navi Z, Etemadi S, Sohrabi Z, Sohrabi N, et al. Seroprevalence of Toxoplasma gondii and Toxocara spp. infections among pregnant women with and without previous abortions in the west of Iran. J Obstet Gynaecol Res. 2020;46:382-8.

20. Mizgajska-Wiktor H, Jarosz W, Fogt-Wyrwas R, Drzewiecka A. Distribution and dynamics of soil contamination with Toxocara canis and Toxocara cati eggs in Poland and prevention measures proposed after 20 years of study. Vet Parasitol. 2017;234:1-9.

21. Habluetzel A, Traldi G, Ruggieri S, Attili AR, Scuppa P, Marchetti R, et al. An estimation of Toxocara canis prevalence in dogs, environmental egg contamination and risk of human infection in the Marche region of Italy. Vet Parasitol. 2003;113:243-52.

22. Sariego I, Kanobana K, Junco R, Vereecken K, Nuñez F, Polman K, et al. Frequency of antibodies to Toxocara in Cuban schoolchildren. Trop Med Int Health. 2012;17:711-4.

23. Mejia R, Seco-Hidalgo V, Garcia-Ramon D, Calderón E, Lopez A, Cooper PJ. Detection of enteric parasite DNA in household and bed dust samples: potential for infection transmission. Parasit Vectors. 2020;13:1-5. 
24. Martínez I, Vázquez O, Romero R, Gutiérrez E, Chasin O. The prevalence of Toxocara cati in domestic cats in Mexico City. Elsevier. 2003;114:43-9.

25. Wolfe A, Wright IP. Human toxocariasis and direct contact with dogs. Vet Rec. 2003;152:419-22.

26. Aydenizöz-Özkayhan M, Yağcı BB, Erat S. The investigation of Toxocara canis eggs in coats of different dog breeds as a potential transmission route in human toxocariasis. Vet Parasitol. 2008:152:94-100.

27. Roddie G, Stafford P, Holland C, Wolfe A. Contamination of dog hair with eggs of Toxocara canis. Vet Parasitol. 2008;152:85-93.
28. Overgaauw P, Nederland V. Aspects of Toxocara epidemiology: toxocarosis in dogs and cats. Crit Rev Microbiol. 1997;23:233-51.

\section{Publisher's Note}

Springer Nature remains neutral with regard to jurisdictional claims in published maps and institutional affiliations.
Ready to submit your research? Choose BMC and benefit from:

- fast, convenient online submission

- thorough peer review by experienced researchers in your field

- rapid publication on acceptance

- support for research data, including large and complex data types

- gold Open Access which fosters wider collaboration and increased citations

- maximum visibility for your research: over $100 \mathrm{M}$ website views per year

At BMC, research is always in progress.

Learn more biomedcentral.com/submissions 